\section{A produção do conhecimento científico e as políticas de saúde pública: reflexões a partir da ocorrência da filariose na cidade do Recife, Pernambuco, Brasil}

\author{
Scientific knowledge and public health policies: \\ reflections on the occurrence of filariasis \\ in Recife, Pernambuco, Brazil
}

Cynthia Braga 1,2

Maria de Fátima P. Militão Albuquerque 1,3 Heloísa Mendonça de Morais 4

\footnotetext{
1 Centro de Pesquisas Aggeu Magalhães, Fundação Oswaldo Cruz, Recife, Brasil. 2 Instituto Materno Infantil de Pernambuco, Recife, Brasil. 3 Departamento de Medicina Clínica, Universidade Federal de Pernambuco, Recife, Brasil. 4 Departamento de Medicina Social, Universidade Federal de Pernambuco, Recife, Brasil.

Correspondência Cynthia Braga Centro de Pesquisas Aggeu Magalhães, Fundação Oswaldo Cruz. Av. Professor Moraes Rego s/n, Recife, PE 50670-420, Brasil. braga@cpqam.fiocruz.br
}

\begin{abstract}
This article discusses the relationship between the production of scientific knowledge on filariasis in Brazil and health policy-making and implementation related to control of the disease. The study presents a review of scientific output on filariasis from the creation of the Program to Combat Filariasis in the mid-20th century until the creation of the Unified National Health System (SUS) within the framework of decentralized control of endemic diseases in the country. The focus on empirical observations in Recife, a city where filariasis is present, highlights the relevant institutional processes and the role of various players. The hypothesis is that in the first half of the 20th century there was a lack of scientific knowledge and technological development to control the problem, but that after these advances were obtained, new requirements of a political nature came into play and acted as determinants for persistence of the endemic in the city.
\end{abstract}

Lymphatic Filariasis; Health Policy; Review Literature
O conhecimento científico e a implementação de políticas de saúde

Decorrido mais de meio século de atividades do Programa de Controle da Filariose no Brasil, a endemia ainda persiste como problema de saúde pública, a despeito dos avanços científicos alcançados quanto às estratégias para seu controle.

Esse fato suscita alguns questionamentos a respeito dos caminhos percorridos desde a identificação de um evento enquanto problema de saúde pública, até a elaboração e a execução das políticas públicas voltadas para sua resolução.

O processo de formulação e implementação das políticas de saúde acontece em uma arena pública estando sujeito a um conjunto muito diverso de determinantes. Assim, transformar conhecimentos científicos em políticas de saúde constitui uma ação extremamente complexa, na qual muitas circunstâncias estão envolvidas, tais como, conjuntura social e política, interesses políticos, poder de mobilização e negociação da população, prioridades sociais e alocação de recursos, questões culturais, preconceitos, ou a vontade e o poder de decisão do formulador ou dos formuladores das políticas 1,2,3. Por tudo isso, Sommer 3 afirma que em muitas ocasiões as evidências científicas em saúde pública exercem pouca ou nenhuma influência no processo. 
Para Orosz 4 , compreender a intrincada e multifacetada relação entre a produção de conhecimento e a elaboração, implementação e avaliação de uma política de saúde específica, requer analisá-la sob diferentes dimensões. Ou seja, verificar quais contextos políticos, culturais e sócio-econômicos favoreceram ou mesmo inibiram sua elaboração, qual a influência e o interesse dos diferentes atores envolvidos, entre eles, a comunidade acadêmica e os formuladores e implementadores das políticas e, finalmente, que impacto terá a política na população.

Especificamente no que se refere às relações entre a comunidade científica e os formuladores das políticas, algumas dificuldades freqüentemente surgem na interpretação de um problema ou na tentativa de solucioná-lo, muitas dessas conseqüentes à lógica ou padrões de referência distintos inerentes a cada um desses grupos 2,4.

Por outro lado, membros de um mesmo grupo podem ter opiniões divergentes a respeito de um problema ou de uma política de saúde, tanto no que se refere à comunidade científica, quanto aos planejadores. Em ambos os casos, alcançar o consenso quanto à solução de um determinado problema nem sempre é uma tarefa fácil 3,4 .

Além disso, alguns cientistas tendem a acreditar que argumentos lógicos e científicos são suficientes para guiar as políticas de saúde e que as mesmas deveriam ser formuladas mediante um processo racional que se moveria a partir da coleta dos dados, sua interpretação, até chegar a um consenso científico 4. Para eles, as evidências falariam por si só e seriam suficientes para a formulação e implementação das políticas de intervenção sobre os processos saúde-doença. Nesses casos, o que lhes falta é uma compreensão sobre os obstáculos inerentes à implementação das políticas.

Por outro lado, muitas políticas são formuladas sem uma clara identificação do problema ou se fundamentam em argumentos considerados incorretos pelos cientistas 5 . Em certas situações, resultados de pesquisas altamente consistentes podem não ser suficientes para orientar ações de saúde pública. Por exemplo, mais de trinta anos depois do primeiro relatório a respeito das conseqüências do tabagismo para a saúde e sete anos depois de ficar estabelecido que a nicotina causa dependência é que o cigarro começou a ser considerado um instrumento de disseminação da nicotina 1 .

Quanto aos formuladores das políticas, podem se ver enredados em algumas armadilhas. Por vezes, esperam respostas científicas preci- sas sobre problemas multifacetados em sua determinação e mutantes em seus efeitos. Em outras circunstâncias, os desafios de realidades por demais complexas os conduzem a posturas paralisantes frente à urgência das demandas impostas aos serviços de saúde.

De fato, o que caberia entender é o processo de decisão política com base na análise das distintas conjunturas nas quais o mesmo se realiza, esclarecendo que essa análise estará sempre voltada para um determinado tipo de intervenção. A percepção da complexidade do problema, dos interesses envolvidos no seu equacionamento (ou não), da imprevisibilidade das soluções propostas, são momentos dinâmicos do processo de tomada de decisão que devem ser sempre considerados.

Embora pouco esclarecido, o processo no qual a decisão política é mobilizada pode ser influenciado tanto pela posição dos políticos ou legisladores, quanto pela pressão política exercida pela população 1 .

Por sua vez, o interesse da população em um determinado problema de saúde está intimamente relacionado ao contexto social, político e cultural no qual ele ocorre, ao grau de conhecimento da população sobre o mesmo, de seu acesso à educação e à renda e ao impacto so cioeconômico do agravo e, por fim, à inserção social do segmento populacional atingido pelo problema e a quem se destinam as políticas.

Especificamente, no que se refere ao problema da filariose bancroftiana, cujos principais determinantes são constituídos pela pobreza e precárias condições de vida a que está submetido considerável segmento da população urbana brasileira 6 , o poder de pressão social exercido por esses atores, considerando seu próprio nível de inserção na estrutura e dinâmica da sociedade, decerto, não tem sido suficiente para mobilizar a vontade política necessária à incorporação do conhecimento produzido às políticas voltadas ao controle da endemia.

Com base nestas considerações, este artigo pretende analisar como se tem dado a articulação entre a produção de um conhecimento específico e a formulação e implementação de políticas dirigidas à solução do problema filariose bancroftiana. A hipótese orientadora da discussão é que se num determinado contexto faltavam o conhecimento científico e o desenvolvimento tecnológico, a seguir, quando esses avanços são disponibilizados, outros requerimentos, agora do campo da política, irão se impor, atuando como determinantes da persistência da endemia na cidade. 


\section{Políticas de saúde, desenvolvimento institucional e a produção do conhecimento em filariose}

No Brasil, a questão de quando e por que a saúde se torna objeto de interesse público e de iniciativas políticas e, ademais, que condições tornaram possível transformar o interesse público em políticas públicas, tem ocupado alguns autores 7,8,9,10. Se uma corrente de pensamento aponta na direção de uma relação causal direta entre interesses econômicos e criação das políticas de saúde pública, do que são exemplos os trabalhos de Braga \& Paula 9 e de Singer et al. 10, outros autores sugerem introduzir a necessidade da construção de uma ideologia da nacionalidade e a questão da constituição do Estado como elementos imprescindíveis ao mais completo entendimento do problema 7,8. Assim, lembrando que entre 1898 e 1910 a cidade do Recife esteve incluída entre aquelas que foram alvos das primeiras campanhas sanitárias realizadas no país, Iyda 8 comenta que as campanhas tiveram a função de estabelecer a organização da atividade estatal, formando inclusive intelectuais aptos para dirigi-la. De um modo ou de outro, os registros historiográficos apontam para o "surgimento já na Primeira República de aparatos públicos, nos planos nacional e estadual, encarregados de executar políticas de saúde e de saneamento" 7 (p. 22).

O modelo sanitarista-campanhista que foi implementado por toda a primeira metade do século XX, na verdade padecia de constrangimentos decisivos: em decorrência do escasso conhecimento científico e da restrita base econômica do Estado brasileiro para fazer frente à montagem da infra-estrutura dos núcleos urbanos nascentes, eram muito reduzidas as possibilidades de intervenções eficazes sobre os agravos endêmicos prevalecentes naquele contexto.

Ainda que o desenvolvimentismo enquanto possibilidade para a alavancagem do projeto econômico do país viesse se consolidando desde os anos trinta, foi na década seguinte que se fizeram sentir seus resultados mais evidentes. Desse modo, o processo de modernização econômica e institucional que se seguiu à promulgação da Constituição de 1946, trouxe consigo a criação dos serviços nacionais de controle de várias doenças endêmicas, como a malária, a febre amarela e a tuberculose. É importante ressaltar que as atividades de controle a partir de então desenvolvidas, se diferenciavam das campanhas anteriores em alguns aspectos, tais como, a estrutura organizacional, o caráter permanente das ações e a cobertura para todo o território nacional 11,12.
Em relação à filariose, data de 1951 o início das atividades da campanha 13. Sabe-se que até então se dispunha de poucos registros sobre o comportamento da endemia no Brasil. Após os primeiros relatos de casos de filariose em 1868 14, vários artigos foram publicados até os anos cinqüenta 15 .

Em todo o período, alguns estudos populacionais de importância foram realizados nas cidades de Salvador, Belém e Maceió, demonstrando se constituírem aquelas localidades em importantes focos da endemia 16,17,18. Todavia, as informações existentes não permitiam avaliar a dimensão do problema em todo o país 18 .

Coube, então, ao Dr. René Rachou, coordenador do Serviço Nacional de Malária (SNM), a missão de elaborar e executar os planos iniciais da Campanha, cujo principal objetivo foi realizar estudos sobre a ocorrência de filariose no país 13,15 .

Os inquéritos filariais realizados permitiram identificar portadores de microfilaremia em setenta e duas localidades $(28,7 \%)$, porém apenas onze foram reconhecidas como áreas de transmissão: Manaus, no Estado do Amazonas; Belém, no Pará; São Luis, no Maranhão; Recife, em Pernambuco; Maceió, em Alagoas; Salvador e Castro Alves, na Bahia; Florianópolis, Ponta Grossa e Barra, em Santa Catarina e Porto Alegre, no Rio Grande do Sul. Entre esses, os de Belém e do Recife foram considerados os de maior importância médico-sanitária 19.

Desse trabalho resultou um vasto número de publicações em periódicos nacionais, que no período vieram a representar parcela expressiva da literatura científica sobre o assunto. Em 1954, por exemplo, entre trinta e dois artigos publicados sobre o tema, trinta se referiam a resultados de inquéritos nas diversas localidades pesquisadas. A maior parte das publicações da época teve sua origem nos próprios serviços responsáveis pelo controle da filariose, sendo ainda bastante incipiente a contribuição dos centros acadêmicos nacionais. Registra-se a expressiva e valiosa contribuição de René Rachou e colaboradores, nas décadas de 50 e 60 18,19,20,21,22,23.

Com base nesses levantamentos realizados pelo SNM, foram propostas diversas medidas de controle da endemia, objetivando o combate ao agente etiológico, a Wuchereria bancrofti, mediante o tratamento com a dietilcarbamazina (DEC), e o controle do mosquito transmissor, o Culex quinquefasciatus, por meio da eliminação dos criadouros reais e potenciais com inseticidas clorados 13 .

A partir de 1955, foram iniciados os inquéritos de avaliação nas áreas endêmicas, objeti- 
vando identificar e tratar os portadores de microfilaremia, além de verificar, por meio do acompanhamento dos níveis de endemicidade, a efetividade das ações desenvolvidas 13 .

$\mathrm{O}$ alto custo dos inseticidas clorados, aliado à sua ineficácia, comprovada por estudos desenvolvidos pelo SNM 20,24, levaram ao abandono das medidas de combate ao vetor, ainda durante a década de 50. Assim, a partir de 1956, as atividades da Campanha de Filariose se restringiram à identificação e tratamento dos microfilarêmicos e ao desenvolvimento de ações de educação sanitária 25 .

Em 1956, a criação do Departamento Nacional de Endemias Rurais (DNERu), absorveu os Serviços Nacionais de Febre Amarela, Malária e Peste e centralizou todos os serviços de combate às endemias, inclusive os da filariose 18. Porém, a limitação das verbas destinadas às áreas sociais - marca, inclusive, do desenvolvimentismo nacionalista dos anos JK - que caracterizou a década de 50 , acabou por comprometer a eficiência de alguns programas de controle das endemias, entre eles, o da filariose. Além disso, a manutenção de práticas ultrapassadas, a carência de investimentos em pesquisas de novas estratégias de intervenção, além da falta de investimentos na formação de recursos humanos contribuíram para a ineficiência do programa 26,27 .

Malgrado todas as limitações para o controle da endemia, ao final da década de 60, um relatório da Organização Mundial da Saúde (OMS), apontava uma redução das áreas de transmissão, identificadas na década anterior, de onze para oito localidades: Salvador e Castro Alves, na Bahia; Belém, Soiré e Vigia, no Pará; Recife e Jaboatão, em Pernambuco 28.

Mediante uma reforma do Ministério da Saúde (MS) em 1970, os Serviços de Erradicação da Malária (CEM), Varíola (CEV) e o DNERu se fundem em uma única instituição, agora denominada Superintendência de Campanhas de Saúde Pública (SUCAM). Embora tenha havido uma elevação dos recursos financeiros para o combate às endemias, a estrutura organizacional e administrativa do novo órgão conservou a mesma lógica centralizadora e verticalizada que caracterizava as ações de controle de endemias no país 9,12.

No campo científico, ocorreram importantes avanços no conhecimento sobre aspectos ligados à biologia do parasita, transmissão, características clínicas e tratamento da filariose linfática 29,30,31. Todavia, esses aportes não permitiram a elaboração de novas estratégias de intervenção capazes de barrar a tendência de expansão da endemia em muitos países, sobre- tudo nos menos desenvolvidos. Havia, portanto, uma grande necessidade de desenvolver novos estudos para avaliar a magnitude mundial do problema e, ao mesmo tempo, aprofundar o conhecimento de aspectos ligados à transmissão da infecção. Igualmente, com vistas ao aprimoramento das ações de controle sobre a doença, fazia-se necessário pesquisar novas formas de combate aos vetores, novas estratégias de tratamento - mais efetivas e menos danosas à saúde da população - e meios diagnósticos mais sensíveis 31,32 .

A partir de 1975, a atenção e o interesse pelo problema da filariose, no mundo, se renovaram com a criação do Programa Especial Internacional para Pesquisa e Treinamento em Doenças Tropicais (TDR), pela OMS, mediante financiamento do Banco Mundial e do Programa de Desenvolvimento da Organização das Nações Unidas (ONU), para apoiar a pesquisa de novas estratégias de controle de doenças endêmicas, treinar cientistas e fortalecer as instituições de pesquisa nos países menos desenvolvidos 33

No início da década de 80, publicações do MS atestavam o sucesso das ações do Programa de Controle da Filariose em vários Estados 34,35. Todavia, no Recife, um estudo epidemiológico, realizado por Morais 29, alertou para o fato da redução encontrada nos níveis endêmicos para o município como um todo, não ser mantida, quando os mesmos dados eram desagregados segundo os diferentes distritos da cidade. Igualmente, a autora identificou uma série de falhas no Programa, entre as quais, a inadequação no horário da coleta, assim como a quantidade insuficiente e imprecisa das amostras de sangue utilizadas na feitura das lâminas pela técnica da gota espessa, que poderiam ter contribuído para subdimensionar o problema. Além disso, a falta de informação sobre o porcentual de adesão ao tratamento e o largo intervalo entre os inquéritos periódicos constituíam sérias limitações ao Programa.

Em 1983, um relatório do Comitê de Especialistas em Filariose da OMS, do mesmo modo, identificava falhas nos programas de controle desenvolvidos nos diversos países endêmicos e apontava a estrutura verticalizada como uma das causas da expansão da endemia, no mundo. Em face dessa constatação, propunha a elaboração de novos modelos de intervenção, integrados à rede de atenção primária à saúde e a intensificação das pesquisas sobre aspectos da transmissão, tratamento e controle da doença 36 .

Em 1986, a VIII Conferência Nacional de Saúde, marco na história da saúde pública bra- 
sileira, propôs a criação do Sistema Único de Saúde (SUS), segundo os princípios da descentralização, eqüidade e integralidade das ações 37. A nova proposta deveria representar mudanças no modelo assistencial vigente, voltado sobretudo ao tratamento individual dos doentes, para um modelo fundamentado nas ações de caráter preventivo e coletivo.

Por outro lado, as mudanças institucionais que ocorreram na Fundação Oswaldo Cruz (FIOCRUZ) nesse mesmo período, precisamente a partir de 1985, quando o sanitarista Sérgio Arouca assume sua presidência, viriam repercutir intensamente nos rumos do Centro de Pesquisas Aggeu Magalhães (CPqAM), unidade da FIOCRUZ localizada na cidade do Recife. Desde 1986, quando o pesquisador André Furtado passa a dirigir essa instituição, verifica-se um impulso notável em suas atividades de pesquisa. Esses fatos bem podem ilustrar uma questão previamente comentada neste artigo e que se refere à função facilitadora para o avanço do conhecimento/implementação de políticas de saúde, em conjunturas particulares. Ou seja, o processo de desenvolvimento institucional ocorrido no CPqAM fez avançar o conhecimento científico sobre a filariose bancroftiana. Mas, atuando como causa, configurouse igualmente enquanto efeito, pois que os financiamentos obtidos para a pesquisa voltada à endemia, terminaram se constituindo no fomento mais significativo para a alavancagem técnico-científica da instituição nos últimos quinze anos.

Foi assim que, em 1986, instituiu-se o Programa de Pesquisas em Filariose que visava a realização de investigações que avaliassem a situação da filariose linfática na região e esclarecessem aspectos de sua transmissão, além de estimular a elaboração de projetos de pesquisa básica e aplicada sobre a doença e a prestação de assistência especializada à população 38,39.

Em seguida, durante o I Simpósio Nacional de Filariose (1987), confirmou-se não só a persistência da endemia no Recife, como também se constatou a existência de diversas áreas de transmissão nos municípios de Olinda e Jaboatão dos Guararapes, ambos situados na Região Metropolitana. Diante disso, recomendou-se ao MS estender a cobertura do Programa de Controle desenvolvido pela SUCAM àqueles municípios 38,39.

Contribuições relevantes foram dadas ao maior conhecimento do comportamento da endemia na região. Registram-se aqui os estudos realizados por Dreyer e colaboradores, que permitiram determinar o horário de pico da microfilaremia no Recife, situado entre 23:00 e
01:00, o que contribuiu para a definição do horário apropriado para a coleta de sangue para o exame parasitológico 38 . Igualmente, o uso inapropriado de anti-histamínicos como coadjuvante ao tratamento antiparasitário com a DEC, na rotina dos serviços especializados, foi oportunamente observado por Dreyer \& Andrade 40, alertando para uma prática inadequada que se reproduzia há décadas, sem ser questionada.

De todo o exposto pode-se afirmar que os resultados das pesquisas desenvolvidas no decorrer dos anos oitenta contribuíram, de alguma forma, para mudanças pontuais nas ações do Programa. Ao final da década de 80, a SUCAM passou a adotar procedimentos metodológicos distintos dos anteriormente utilizados na aplicação da técnica da gota espessa, resultando em uma elevação da positividade das lâminas nas estatísticas oficiais já a partir de 1989 6. Da mesma forma, os serviços passaram a não mais utilizar a associação de anti-histamínicos e DEC. Também, a partir de 1989, as atividades do Programa, que permaneciam limitadas à identificação e tratamento dos microfilarêmicos, mediante inquéritos porta a porta e a demanda espontânea aos serviços, foram expandidas a outros municípios da Região Metropolitana do Recife 41 .

Todavia, o enfoque da produção científica sobre o tema, até aquele momento, não oferecia subsídios para possíveis mudanças do modelo de atenção prestado.

\section{A descentralização do controle das endemias e as novas propostas de intervenção sobre a filariose}

A Lei Orgânica da Saúde promulgada em 1990, já no contexto das transformações decorrentes da VIII Conferência Nacional de Saúde, estabeleceu as bases do processo de descentralização das ações de saúde, dentre estas, do controle das endemias 37 .

Como parte daquele processo, a SUCAM, a Fundação de Serviços Nacionais de Saúde Pública (FSESP) e o Departamento de Informática do SUS (DATASUS) foram reunidos, em 1991, dando origem à Fundação Nacional de Saúde (FNS), que passou a ser o órgão responsável pelas ações de controle das doenças transmissíveis e de saneamento em âmbito nacional. Nesse período, os recursos destinados ao controle das endemias eram, em sua maioria, originários do Tesouro Nacional, porém, com a progressiva redução dos recursos, outras fontes de financiamento passaram a ser utilizadas. Entre elas, o Banco Mundial, por meio dos Pro- 
jetos de Controle das Doenças Endêmicas no Nordeste (PCDEN) e da Malária na Amazônia (PCMAM) 42.

Em 1992, durante a IX Conferência Nacional de Saúde foi dado mais um passo rumo à descentralização do controle das endemias, quando da aprovação da extinção da FNS e o repasse imediato de suas unidades para os estados e municípios 43 .

Na cidade do Recife, os anos noventa foram particularmente importantes no avanço do conhecimento sobre a filariose, estando o CPqAM sempre à frente do processo de consecução de novos aportes. Por outro lado, abriram-se perspectivas inovadoras de diálogo entre os pesquisadores e os serviços estaduais e municipais de saúde. Assim, entre 1990 e 1993, foram desenvolvidos vários estudos clínicos e epidemiológicos, alguns deles contando com a colaboração de consultores internacionais, ainda que se questionasse a dimensão da endemia na cidade.

Entre os anos de 1991 e 1992, com o apoio financeiro do programa TDR da OMS, da Fundação de Amparo à Ciência e Tecnologia do Estado de Pernambuco (FACEPE) e do CNPq, o CPqAM realizou novo inquérito de filariose no Recife 44 . Com base nos resultados apresentados por Morais 33, o estudo foi direcionado para áreas mais carentes de infra-estrutura do município, tendo sido investigadas vinte e duas favelas. A prevalência global de microfilaremia encontrada foi de $6,5 \%$, nível de endemicidade semelhante ao observado por Rachou, na década de 50. Em algumas localidades, a microfilaremia atingiu valores acima de $10 \%$, sendo as respectivas áreas consideradas de alta endemicidade, segundo critério da OMS 45. Constatouse, desse modo, que a filariose se mantinha como um grave problema de saúde pública no Recife e que as ações desenvolvidas pelo Programa desde a década de 50 , continuavam sendo ineficazes.

Nesse período, dados da literatura mundial atestavam a eficácia do tratamento com doses baixas e esparsas de DEC, administrada a toda a população 46,47,48,49,50. As evidências epidemiológicas da eficácia desse esquema terapêutico com doses baixas de DEC, administradas periodicamente em intervalos regulares, significaram um grande avanço para os programas de controle no sentido de diminuir em muito o abandono do tratamento 48 . No âmbito do controle do vetor, algumas novas medidas também haviam sido testadas com sucesso 51,52. Esses resultados motivaram pesquisadores do CPqAM a realizar um estudo visando a testar a efetividade e viabilidade das estratégias do tratamento em massa com DEC e do controle integrado do vetor, em duas áreas endêmicas do Recife $53,54,55,56$. A pesquisa realizou-se entre $1990 \mathrm{e}$ 1993 e, mais uma vez, contou com o apoio financeiro do programa TDR e da FACEPE, e institucional da FNS e das Secretarias Estadual e Municipal de Saúde. Foram estudadas duas comunidades com níveis de endemicidade e condições ambientais similares. Após três ciclos de tratamento com DEC, em doses baixas, mais de $50 \%$ dos microfilarêmicos tiveram seus exames negativados. Além disso, houve uma redução de $96 \%$ da carga parasitária na população 53 . Em uma das comunidades na qual, além do tratamento em massa, realizou-se o controle integrado do vetor, a densidade populacional do Culex caiu de 60 a 120 mosquitos/quarto/ noite para cerca de 10 mosquitos/quarto/noite 56. Do mesmo modo, o índice de infecção vetorial que era de $3 \%$ antes do início das ações de controle, foi reduzido a zero, sugerindo a interrupção do ciclo de transmissão. A estratégia de tratamento praticamente não produziu efeitos colaterais e a adesão da comunidade foi maior que $80 \% 54$. Tais resultados significavam que as ações de tratamento em massa e do controle integrado de vetores se constituíam em uma possibilidade efetiva para o controle da endemia.

Progressivamente, firmava-se o papel do CPqAM enquanto instituição dedicada à pesquisa em filariose. Algumas das pesquisas ali realizadas obtiveram reconhecimento internacional, contribuindo para o esclarecimento de aspectos relativos à patogenia e ao diagnóstico da doença 57,58,59,60,61,62,63,64,65,66,67,68,69. Ademais, ensaios clínicos testando novas drogas e esquemas terapêuticos concorreram para o maior conhecimento sobre o tratamento da doença 70,71,72,73,74,75,76,77,78. Na área de controle do vetor, várias pesquisas forneceram importantes subsídios ao aprimoramento das intervenções $66,79,80,81,82,83,84,85$. No campo da epidemiologia, estudos desenvolvidos durante a década de 90 , permitiram um maior entendimento sobre o processo de determinação da doença 6 e possibilitaram um maior conhecimento acerca da ocorrência da endemia na região 44,86,87,88,89,90.

Finalmente, corroborando o que os pesquisadores locais haviam concluído, adveio do cenário científico internacional um importante pronunciamento. Os expertos da Força Tarefa Internacional para Erradicação de Doenças grupo de cientistas constituído para avaliar o potencial de erradicação das doenças transmissíveis - considerando as particularidades biológicas do parasita, características de transmissão e os grandes avanços obtidos nas estratégias de intervenção, declararam a filariose 
como uma das seis doenças passíveis de serem erradicadas no mundo 91 . Na mesma direção, mais recentemente, pesquisadores do TDR/ OMS incluíram a filariose linfática no grupo das doenças de menor prioridade em pesquisas, considerado o declínio no número de casos e a disponibilidade de estratégias de controle com comprovada efetividade 92 .

No Recife, a constatação de que as medidas de controle da endemia continuavam se mostrando ineficazes, e mais, um contexto nacional no qual eram discutidas as diretrizes e a implementação do SUS, motivaram a elaboração de um modelo de intervenção de base territorial e estruturado com base nos serviços de saúde 93. A nova proposta teve por princípios a descentralização do controle das endemias e a análise epidemiológica dos problemas de saúde, direcionada para os seus vários níveis de determinação. No modelo, o foco das ações foi deslocado dos indivíduos microfilarêmicos para a identificação das áreas de maior risco de transmissão da infecção. A estratificação do espaço urbano deveria ser feita mediante a utilização de um indicador composto construído baseando-se em informações acerca do território, todas, teórico e empiricamente, relacionadas à transmissão da $W$. bancrofti. O indicador, assim construído, deveria ser sensível o suficiente para refletir diferenças nas condições de vida dos grupos populacionais no que diz respeito às habitações, presença de equipamentos de infra-estrutura urbana e densidade populacional, expressando, conseqüentemente, diferenças no risco de contrair a filariose. Assim, o tratamento em massa de grupos populacionais e o controle integrado de vetores estaria indicado em áreas de maior risco de transmissão e com altos níveis endêmicos, mantendo-se a indicação de tratamento seletivo para os indivíduos infectados, residentes em áreas de baixo risco de transmissão 93 .

Procurava-se, desse modo, garantir a eqüidade em relação às ações de controle, identificando grupos prioritários para a intervenção e reorientando as práticas de saúde para a dimensão do coletivo.

Adicionalmente, com base nos avanços das técnicas computacionais de análise de dados e espacialização da ocorrência dos eventos de saúde, um outro estudo foi desenvolvido para avaliar o indicador de risco ambiental que compunha o modelo de intervenção. Assim, em um município da Região Metropolitana do Recife, encontrou-se que nos dois estratos intra-urbanos caracterizados pelo indicador como de mais alto risco de transmissão, residiam $85,7 \%$ dos indivíduos microfilarêmicos 94 . Os autores concluíram que a alta sensibilidade demonstrada pelo indicador para predizer os locais de ocorrência da maioria dos casos de filariose justificaria sua utilização no planejamento e na execução das ações de controle.

Reuniam-se, assim, as condições ideais para o redirecionamento da intervenção sobre a endemia no Recife. Por um lado, os estudos comprovavam a efetividade do tratamento em massa de populações endêmicas com doses baixas e repetidas de DEC, associado ao controle de vetores para o controle da filariose. Por outro, um modelo de intervenção de base territorial, formulado baseando-se em um novo referencial teórico e metodológico, ficava estabelecido e detalhado em suas questões operacionais. A validação do indicador, capaz de predizer áreas com alto risco ambiental de transmissão da parasitose, significava que não seria mais necessário realizar inquéritos parasitológicos repetidos para a identificação de grupos prioritários para a intervenção.

Quanto à política de controle da filariose, faz-se oportuno lembrar que na segunda metade dos anos noventa, o MS traz à tona o problema em um contexto no qual a OMS propunha a eliminação da endemia em nível mundial 95. Simultaneamente, no Estado de Pernambuco, tomava corpo a discussão de um plano nacional de intervenção, mediante a participação de técnicos dos serviços estaduais e municipais de saúde, inclusive dos demais estados atingidos pela endemia, de membros da comunidade científica, além de consultores da OMS 95. A proposta finalmente elaborada chega ao Conselho Nacional de Saúde (CNS), o qual terminou por recomendar ao MS a implantação de um Plano Nacional de Eliminação da Filariose Linfática (PNEFL). Quanto ao plano estadual, resultou de um trabalho de cooperação técnica desenvolvido entre o Estado e municípios endêmicos da Região Metropolitana do Recife, a FNS/MS e a comunidade científica local, ressaltando-se sua fundamentação nos pressupostos teórico-metodológicos do modelo de intervenção anteriormente proposto. Caracterizava-se assim, tanto no âmbito local, quanto no nacional, um intercâmbio de saberes e experiências historicamente pouco exercitado na formulação das políticas de controle das endemias nacionais.

Finalmente, entre 1998 e 2000, com base em vários inquéritos realizados nos municípios endêmicos do país, pode-se concluir que a filariose ainda se constitui em um problema de saúde pública. Ainda que os níveis de endemicidade sejam diferenciados, alguns apresentando tendência de queda acentuada 95 , inte- 
ressa ressaltar a gravidade do problema no Estado de Pernambuco, em particular na cidade do Recife e em alguns municípios de sua Região Metropolitana 94,96,97. A tal ponto que inquérito muito recente demonstrou o acometimento de indivíduos jovens, indicativo inquestionável da intensidade da transmissão 97.

Considerando que há duas décadas a endemia era dada como erradicada no país, deve ser ressaltado o papel da comunidade científica em questionar um argumento cujas bases epidemiológicas mostravam-se frágeis e de prover insumos adicionais ao conhecimento até aquela época existente, conforme bem demonstrado no decorrer deste trabalho. Por outro lado, as mudanças radicais que ocorreram na política nacional de saúde constituíram o cenário singular a partir do qual tornou-se possível um outro modelo de intervenção. E, da cooperação entre a comunidade das instituições de pesquisa e a dos serviços, emergiu uma proposta pas- sível de ser finalmente operacionalizada enquanto política.

Restaria, então, indagar quanto aos obstáculos para sua implementação. No conjunto dos atores envolvidos nesse processo - comunidade acadêmica, técnicos dos serviços, agências de fomento do desenvolvimento científico, gestores do sistema de saúde - o vazio se faz sentir em relação à população alvo da intervenção. O fato de a filariose ocorrer em espaços urbanos ocupados majoritariamente pelo segmento da população urbana originário do "circuito inferior da economia" 98, decerto estaria contribuindo para o enfraquecimento da pressão social imprescindível ao processo de definição de prioridades para a decisão política. Trata-se, de outro modo, do problema do controle social, nova categoria introduzida na agenda das políticas de saúde no contexto posterior à criação do SUS.

\section{Resumo}

O artigo discute a relação entre a produção científica sobre a ocorrência da filariose bancroftiana no Brasil e o processo de formulação e implementação da política de saúde voltada ao seu controle. Para tanto, realiza uma revisão da produção do conhecimento e das políticas específicas, no período compreendido desde a criação do Programa de Combate à Filariose, em meados do século passado, até a criação do SUS, no marco da descentralização do controle das endemias. Centrando suas observações empíricas na ambiência da cidade do Recife, espaço urbano no qual ainda prevalece a filariose, ressalta os processos institucionais e destaca o papel dos distintos atores neles envolvidos. Partindo da hipótese de que se até à primeira metade do século vinte faltavam o conhecimento científico e o desenvolvimento tecnológico para o controle do problema, a seguir, quando esses avanços são disponibilizados, outros requerimentos, agora do campo da política, irão se impor, atuando como determinantes da persistência da endemia na cidade.

Filariose Linfática; Política de Saúde; Literatura de Revisão

\section{Colaboradores}

Todas as autoras participaram da redação do artigo. C. Braga e M. F. P. M. Albuquerque realizaram a revisão bibliográfica e elaboração da revisão histórica da filariose no Brasil. H. M. Morais abordou o tema sob a ótica das políticas de saúde e procedeu a revisão do texto.

\section{Referências}

1. Atwood C, Coldiz GA, Kawachi I. From public health science to prevention policy: placing science in its social and political contexts. Am J Public Health 1997; 87:1603-6.

2. Pouvourville G. Public health research: between science and action. Cad Saúde Pública 1999; 15: 889-94.

3. Sommer A. How public health policy is created: scientific process and political reality. Am J Epidemiol 2001; 154 (12 Suppl):4-6.

4. Orosz E. The impact of social science research on health policy. Soc Sci Med 1994; 39:1287-93.

5. Rosenstock L, Lee LJ. Attacks on science: the risk to evidence-based policy. Am J Public Health 2002; 92:14-8.

6. Albuquerque MFPM. Urbanização, favelas e endemia: a produção da filariose no Recife, Brasil. Cad Saúde Pública 1993; 9:487-97.

7. Hochman G. A era do saneamento. São Paulo: Editora Hucitec/Associação Nacional de Pós-graduação e Pesquisa em Ciências Sociais; 1998.

8. Iyda M. Cem anos de saúde pública. A cidadania negada. São Paulo: Editora da Universidade Estadual Paulista; 1994

9. Braga JCS, Paula SG. Saúde e previdência. Estudos de política social. São Paulo: Editora Hucitec/ Centro Brasileiro de Estudos de Saúde; 1986.

10. Singer P, Campos O, Oliveira EM. Prevenir e curar. O controle social através dos serviços de saúde. Rio de Janeiro: Editora Forense Universitária; 1978.

11. Mendes EV. O processo social de distritalização da saúde. In: Mendes EV, organizador. Distrito sanitário: o processo social de mudança das práticas 
sanitárias do Sistema Único de Saúde. Rio de Janeiro: Editora Hucitec/ABRASCO; 1995. p. 93-158.

12. Sabroza PC, Kawa H, Campos WSQ. Doenças transmissíveis: ainda um desafio. In: Minayo MC, organizadora. Os muitos Brasis: saúde e população na década de 80. Rio de Janeiro: Editora Hucitec/ABRASCO; 1995. p. 177-244.

13. Franco ORL, Lima DM. Alguns aspectos das atividades contra a filariose bancroftiana no Brasil. Rev Bras Malariol Doenças Trop 1967; 19:74-89.

14. Wucherer O. Notícia preliminar sobre vermes de uma espécie ainda não descrita, encontrados na urina de doentes de hematúria intertropical no Brasil. Gazeta Médica da Bahia 1868; 3:97-9.

15. Coutinho A, Medeiros Z, Dreyer G. História da filariose linfática em Pernambuco. I. Aspectos epidemiológicos e de controle. Rev Soc Bras Med Trop 1996; 29:607-12.

16. Causey OR, Deane MP, Costa OR, Deane LM. Studies on the incidence and transmission of filaria Wuchereria bancrofti in Belém, Brazil. Am J Hyg 1945; 41:143-9.

17. Pessoa SB, Andrade ZA. Alguns dados sobre a incidência da filária Wuchereria bancrofti na Cidade do Salvador, Bahia. O Hospital 1950; 37:593-8.

18. Rachou RG. Conceito e programa de profilaxia da filariose bancroftiana no Brasil. Rev Bras Malariol Doenças Trop 1960; 12:11-39.

19. Rachou RG. Distribuição geográfica das filarioses humanas no Brasil. Rev Bras Malariol Doenças Trop 1957; 9:79-100.

20. Rachou RG. Algumas manifestações de resistência de comportamento de insetos aos inseticidas no Brasil. Rev Bras Malariol Doenças Trop 1958; 10:277-90

21. Rachou RG, Deane LM. Filarioses humanas no Brasil. Conhecimento atual de sua distribuição e transmissão. Rev Bras Malariol Doenças Trop 1954; 6:377-84.

22. Rachou RG, Lobo AGS, Martins JS. Primeiras investigações do Serviço Nacional de Malária relativas à incidência de filariose no Estado do Paraná. Rev Bras Malariol Doenças Trop 1954; 6:477-9.

23. Rachou RG; Neves HA, Scaff LM. Da possibilidade do emprego do hetrazan de curta duração no combate à bancroftose. Rev Bras Malariol Doenças Trop 1955; 7:37-9.

24. Lima MM, Rachou RG. Resistência do Culex pipiens fatigans em Belém do Pará (Brasil) a inseticidas clorados (DDT e Dieltrin). Rev Bras Malariol Doenças Trop 1959; 12:11-39.

25. Scaff LM, Gueiros ZM. Erradicação da filariose. Rev Bras Malariol Doenças Trop 1969; 21:606-13.

26. Bertolli Filho C. História da saúde pública no Brasil. São Paulo: Editora Ática; 1998.

27. Maciel A, Furtado A, Marzochi KB. Perspectivas da municipalização do controle da filariose linfática na Região Metropolitana do Recife. Cad Saúde Pública 1999; 15:193-203.

28. World Health Organization. Report on the incidence of Wuchereria bancrofti in some countries of South America. Geneva: World Health Organization; 1969.

29. Morais HM. A filariose no Recife - um estudo epidemiológico [Dissertação de Mestrado]. São Paulo: Universidade de São Paulo; 1982.
30. Nelson GS. Filariasis. N Engl J Med 1979; 300: 1136-9.

31. Nelson GS. Issues in filariasis - a century of enquiry and century of failure. Acta Trop 1981; 38 : 197-204.

32. Grove DJ. Selective primary health care: strategies for the control of diseases in the developing world. VII Filariasis. Rev Infect Dis 1983; 5:933-44.

33. Morel CM. Reaching maturity -25 years of TDR. Parasitol Today 2000; 16:2-8.

34. Ministério da Saúde. Controle de endemias em 1983. Brasília: Superintendência de Campanhas de Saúde Pública; 1983.

35. Ministério da Saúde. O controle das endemias (de 1979 a 1984). Brasília: Superintendência de Campanhas de Saúde Pública; 1985.

36. World Health Organization. Lymphatic filariasis. Geneva: World Health Organization; 1984.

37. Oliveira Jr. M. O financiamento da área social e do Sistema Único de Saúde no Brasil. http: / /www. datasus.gov.br/ (acessado em 12/Set/2002).

38. Dreyer G. Filariasis programme - Recife - Brazil. Mem Inst Oswaldo Cruz 1987; 82:359-60.

39. Dreyer G, Medeiros Z. Filariose linfática: ainda um desafio. Ciência Hoje 1990; 12:6-7.

40. Dreyer G, Andrade L. Inappropriateness of the association of diphenhydramine with diethylcarbamazine for the treatment of lymphatic filariasis. J Trop Med Hyg 1989; 92:32-4.

41. Secretaria de Saúde do Estado de Pernambuco. Proposta de intervenção sobre a filariose linfática em Pernambuco. Recife: Diretoria Executiva de Epidemiologia e Vigilância Sanitária, Secretaria de Saúde do Estado de Pernambuco; 1996.

42. Carvalho EMF, Acioli MD. Pesquisa de avaliação do impacto de programas IEC/MC no controle das doenças endêmicas em cinco estados brasileiros - versão Pernambuco. Relatório final. Recife: Ministério da Saúde; 1996.

43. Ministério da Saúde. História da XIX Conferência Nacional de Saúde. http://www.fiocruz.br/histcon ferencias/tema9.html (acessado em 11/Set/2002).

44. Maciel A, Rocha A, Marzochi KB, Medeiros Z, Carvalho $A B$, Regis $L$, et al. Epidemiological study of bancroftian filariasis in Recife, Northeastern Brazil. Mem Inst Oswaldo Cruz 1996; 91:449-55.

45. Organización Mundial de la Salud. Lucha contra la filariasis linfática. Manual para personal sanitário. Geneva: Organización Mundial de la Salud; 1988.

46. Kessel JF. An effective programme for the control of filariasis in Tahiti. Bull World Health Organ 1957; 16:633-44.

47. Laigret J, Kessel JF, Bambridge B, Adams H. Onze ans de chimioprophilaxie par la diéthylcarbamazine de la filariose lymphatique apériodique à Tahiti. Bull World Health Organ 1966; 34:925-38.

48. Hawking F. Diethylcarbamazine and new compounds for the treatment of filariasis. Adv Pharmacol Chemother 1979; 16:129-94.

49. Nathan MB, Hamilton PJ, Monteil S, Tikasingh ES. Bancroftian filariasis in coastal North Trinidad: the effects of mass chemotherapy using spaced doses of diethilcarbamazine citrate on human microfilaraemias and vector infection rates. Trans R Soc Trop Med Hyg 1987; 81:663-8. 
50. Eberhard ML, Lammie PJ, Roberts JM, Lowrie Jr. RC. Effectiveness of spaced doses of diethylcarbamazine citrate for the control of bancroftian filariasis. Trop Med Parasitol 1989; 40:111-3.

51. Nicolas L, Dossou-Yovo J. Differential effects of the Bacillus sphaericus strain 2362 on Culex quinquefasciatus and its competitor Culex cinereus in West Africa. Med Vet Entomol 1987; 1:23-7.

52. Rajagopalan PK, Das SP, Pani SP, Mariappan T, Rajavel AR, Ramaiah KD, et al. Evaluation of the integrated vector control measures on filariasis transmission in Pondicherry. Indian J Med Res 1988; 87:434-9.

53. Furtado AF, Maciel A, Rocha A, Regis L, Braga MC, Ferreira RMR. Controle da filariose na Cidade do Recife: eficácia do tratamento em massa da população, utilizando baixas doses de dietilcarbamazina (DEC). Rev Soc Bras Med Trop 1994; 27 Suppl 1:109.

54. Furtado AF, Regis L, Maciel A, Rocha A, Silva-Filha $\mathrm{MH}$, Braga C, et al. Controle da filariose na Cidade do Recife: tratamento em massa com a dietilcarbamazine (DEC) associado ao controle de vetores. Rev Soc Bras Med Trop 1994; 27 Suppl 1:16.

55. Centro de Pesquisas Aggeu Magalhães. Sucesso no controle da filariose. Informe Fieldlincs 1. Recife: Centro de Pesquisas Aggeu Magalhães, Fundação Oswaldo Cruz; 1992.

56. Regis L, Silva-Filha MH, Oliveira CM, Rios EM, Silva SB, Furtado AF. Integrated control measures against Culex quiquefasciatus, the vector of filariasis in Recife. Mem Inst Oswaldo Cruz 1995; 90: 115-9.

57. Amaral F, Dreyer G, Figueiredo-Silva J, Norões J, Cavalcanti A, Samico SC, et al. Live adult worms detected by ultrasonography in human bancroftian filariasis. Am J Trop Med Hyg 1994; 50:753-4.

58. Dreyer G, Andrade L, Espírito Santo M, Medeiros Z, Moura I, Tenório J, et al. Avaliação do teste de imunofluorescência indireta para o diagnóstico da filariose bancroftiana usando a microfilária de Wuchereria bancrofti como antígeno em RecifePE. Rev Inst Med Trop São Paulo 1991; 33:397-402.

59. Dreyer G, Ottesen E, Galdino A, Andrade L, Rocha A, Medeiros Z, et al. Renal abnormalities in microfilaremic with bancroftian filariasis. Am J Trop Med Hyg 1992; 46:745-51.

60. Dreyer G, Amaral F, Norões J, Medeiros Z, Addiss D. A new tool to assess the adulticidal efficacy in vivo of antifilarial drugs for bancroftian filariasis. Trans R Soc Trop Med Hyg 1995; 89:225-6.

61. Dreyer E, Pimentel A, Beliz F, Galdino E, Moura I, Coutinho A, et al. Studies on the periodicity and intravascular distribution of Wuchereria bancrofti microfilariae in paired samples of capillary and venous blood from Recife, Brazil. Trop Med Int Health 1996; 1:264-72.

62. Dreyer G, Norões J, Rocha A, Addiss D. Detection of living adult Wuchereria bancrofti in a patient with tropical pulmonar eosinophilia. Braz J Med Biol Res 1996; 29:1005-8.

63. Freedman DO, Almeida Filho PJ, Besh S, Maia e Silva MC, Braga C, Maciel A. Lymphoscintigraphic analysis of lymphatic abnormalities in symptomatic and asymptomatic human filariasis. J Infect Dis 1994; 170:927-33.
64. Freedman DO, Almeida Filho PJ, Besh S, Silva MCM, Braga C, Maciel A, et al. Abnormal lymphatic function in presymptomatic bancroftian filariasis. J Infect Dis 1995; 171:997-1001.

65. Freedman DO, Almeida A, Miranda J, Plier DA, Braga C. Field trial of a rapid card test for $W u$ chereria bancrofti. Lancet 1997; 350:1681.

66. Furtado AF, Abath FGC, Regis LN, Gomes YM, Lucena WA, Furtado PB, et al. Improvement and application of a polymerase chain reaction system for detection of Wuchereria bancrofti in Culex quinquefasciatus and human blood samples. Mem Inst Oswaldo Cruz 1997; 92:85-6.

67. Jungmann P, Fiqueiredo-Silva J. Bancroftian lymphadenopathy: a histopathologic study of fiftyeight cases from Northeast Brazil. Am J Trop Med Hyg 1991; 45:325-31.

68. Rocha A, Dreyer G, Poindexter R, Ottesen EA. Syndrome resembling tropical pulmonary eosinophilia but of non-filarial aetiology: serological findings with filarial antigens. Trans R Soc Trop Med Hyg 1995; 89:536-75.

69. Rocha A, Addiss D, Ribeiro ME, Norões J, Baliza M, Medeiros Z, et al. Evaluation of the Og4C3 ELISA in Wuchereria bancrofti infection: infected persons with undetectable or ultra-low microfilarial densities. Trop Med Int Health 1996; 1:85964.

70. Andrade LD, Medeiros Z, Pires ML, Pimentel A, Rocha A, Figueiredo-Silva J, et al. Comparative efficacy of three different diethilcarbamazine regimens in lymphatic filariasis. Trans R Soc Trop Med Hyg 1995; 89:319-21.

71. Coutinho AD, Dreyer G, Medeiros Z, Lopes E, Machado G, Galdino E, et al. Ivermectin treatment of bancroftian filariasis in Recife, Brazil. Am J Trop Med Hyg 1994; 50:339-48.

72. Dreyer G, Pires ML, Andrade LD, Lopes E, Medeiros Z, Tenório J, et al. Tolerance of dethylcarbamazine by microfilaraemic and amicrofilaraemic individuals in and endemic area of bancroftian filariasis. Trans R Soc Trop Med Hyg 1994; 88:232-6.

73. Dreyer G, Coutinho A, Miranda D, Norões J, Rizzo J, Galdino E, et al. Treatment of bancroftian filariasis in Recife, Brazil: a two-year comparative study if the efficacy of single treatment with ivermectin or diethylcarbamazine. Trans R Soc Trop Med Hyg 1995; 89:98-102.

74. Dreyer G, Norões J, Amaral F, Nen A, Medeiros Z, Coutinho A, et al. Direct assessment of the adulticidal efficacy of a single dose of ivermectin in bancroftian filariasis. Trans R Soc Trop Med Hyg 1995; 89:441-3.

75. Dreyer G, Addiss D, Norões J, Amaral F, Rocha A, Coutinho A. Ultrasonographic assessment of the adulticidal efficacy of repeated high-dose ivermectin in bancroftian filariasis. Trop Med Int Health 1996; 1:427-32.

76. Dreyer G, Addiss D, Santos A, Figueiredo-Silva J, Norões J. Direct assessment in vivo of the efficacy of combined single-dose ivermectin and diethylcarbamazine against adult Wuchereria bancrofti. Trans R Soc Trop Med Hyg 1998; 92:219-22.

77. Figueiredo-Silva J, Jungmann P, Norões J, Piessens W, Coutinho A, Brito C, et al. Histological evi- 
dence for the adulticidal effect of the low doses of diethylcarbamazine in bancroftian filariasis. Trans R Soc Trop Med Hyg 1996; 90:192-4.

78. Freedman DO, Bui T, Almeida Filho PJ, Besh S, Silva MCM, Braga C, et al. Lymphoscintigraphic assessment of the effect of diethylcarbamazine treatment on lymphatic damage in human bancroftian filariasis. Am J Trop Med Hyg 1995; 52: 258-61.

79. Nicolas L, Regis LN, Rios EM. Role of exosporium in the stability of Bacillus sphaericus binary toxin. FEMS Microbiol Lett 1994; 124:271-6.

80. Oliveira CMF. Condições ambientais e densidade populacional de Culex quinquefasciatus em áreas urbanas [Tese de Doutorado]. Recife: Universidade Federal de Pernambuco; 1996.

81. Regis LN, Furtado A, Silva-Filha MH, Oliveira CM, Silva SB. The impact of Bacillus sphaericus treatments on Culex quinquefasciatus population in urban areas of Recife, Brazil. In: 7th International Colloquium of Invertebrate Pathology and Microbial Control; Montpellier: Society for Invertebrate Pathology; 1994. p. 254-8.

82. Regis L, Silva SB, Maranhão LMC, Rios EM, Carielli MA. Avaliação da eficácia e viabilidade do uso de larvicida à base de B.sphaericus, produzido localmente, no controle de Culex quinquefasciatus em áreas urbanas. Recife: Centro de Pesquisas Aggeu Magalhães, Fundação Oswaldo Cruz; 1999.

83. Regis L, Oliveira C, Silva-Filha M, Silva S, Maciel A, Furtado A. Efficacy of B.sphaericus in control the filariasis vector Culex quinquefasciatus in urban areas of Olinda, Brazil. Trans R Soc Trop Med Hyg 2000; 94:488-92.

84. Silva-Filha MH, Regis L. Reversal of a low-level resistance to Bacillus sphaericus in a field population of the Southern House mosquito (Diptera: Culicidae) from an urban area of Recife, Brazil. J Econ Entomol 1997; 90:299-303.

85. Silva-Filha M, Regis L, Nielsen-Leroux C, Charles JF. Low level resitance to Bacillus sphaericus in a field-treated population of Culex quinquefasciatus (Diptera: Culicidae). J Econ Entomol 1995; 88: 525-30.

86. Albuquerque MFPM, Marzochi MC, Sabroza PC, Braga MC, Padilha T, Silva MCM, et al. Bancroftian filariasis in two urban areas of Recife, Brazil: pre-control observations on infection and diseases. Trans R Soc Trop Med Hyg 1995; 89:373-7.

87. Albuquerque MFPM, Marzochi MC, Ximenes RA, Braga MC, Silva MCM, Furtado A. Bancroftian filariasis in two urban areas of Recife, Brasil: the role of individual risk factors. Rev Inst Med Trop São Paulo 1995; 37:225-33.
88. Braga C, Albuquerque MFM, Schindler HC, Rezende A, Maciel A, Silva MCM, et al. Perfil epidemiológico da filariose linfática em crianças residentes em áreas endêmicas. J Pediatr (Rio J) 1997; 73:95-100.

89. Braga C, Albuquerque MFM, Schindler HC, Silva MRF, Maciel A, Furtado AF, et al. Risk factors for the occurrence of bancroftian filariasis infection in children living in endemic areas of Northeast Brazil. J Trop Pediatr 1998; 44:87-91.

90. Medeiros Z, Gomes J, Beliz F, Coutinho A, Dreyer P, Dreyer G. Screening of army soldiers for $W u$ chereria bancrofti infection in the Metropolitan Recife Region, Brazil: implications for epidemiological surveillance. Trop Med Int Health 1999; 4:499-505.

91. Centers for Disease Control and Prevention. Recommendations of the International Task Force for Disease Eradication. MMWR 1993; 42:1-38.

92. Remme JHF, Blas E, Chitsulo L, Desjeux PMP, Engers HD, Kanyok TP, et al. Strategic emphases for tropical diseases research: a TDR perspective. Trends Parasitol 2002; 18:421-6.

93. Albuquerque MFPM, Morais, HMM. Decentralization of endemic disease control: an intervention model for combating bancroftian filariasis. Rev Panam Salud Pública 1997; 1:155-63.

94. Braga C, Ximenes RA, Albuquerque M, Souza WV, Miranda J, Brayner F, et al. Avaliação de um indicador socioambiental utilizado no rastreamento de áreas de transmissão de filariose linfática em espaços urbanos. Cad Saúde Pública 2001; 17: 1211-8.

95. Ministério da Saúde. Relatório: reunião de avaliação do Programa Nacional de Eliminação da Filariose Linfática. Recife: Fundação Nacional de Saúde; 2000

96. Secretaria de Saúde do Recife. Programa de Saúde Ambiental. Subprograma de Controle da Filariose. Recife: Diretoria de Epidemiologia e Vigilância à Saúde, Secretaria de Saúde do Recife; 2002.

97. Bonfim C, Lessa F, Oliveira C, Evangelista MJ, Espírito-Santo ME, Meireles E, et al. Situação da filariose bancroftiana na Região Metropolitana do Recife: estudo em uma área endêmica no Município de Jaboatão dos Guararapes, Pernambuco, Brasil. Cad Saúde Pública 2003; 19:1497-505.

98. Santos M. O espaço dividido: os dois circuitos da economia urbana dos países subdesenvolvidos. Rio de Janeiro: Livraria Francisco Alves; 1979.

Recebido em 07/Out/2003

Aprovado em 30/Out/2003 